\title{
Symmetries shape the current in ratchets induced by a biharmonic driving force
}

\author{
Niurka R. Quintero, ${ }^{1, *}$ José A. Cuesta, ${ }^{2, \dagger}$ and Renato Alvarez-Nodarse ${ }^{3, *}$ \\ ${ }^{1}$ Departamento de Física Aplicada I, E.U.P., Universidad de Sevilla, Virgen de África 7, E-41011 Sevilla, Spain \\ ${ }^{2}$ Grupo Interdisciplinar de Sistemas Complejos (GISC), Departamento de Matemáticas, Universidad Carlos III de Madrid, \\ Avda. de la Universidad 30, Leganés, E-28911 Madrid, Spain \\ ${ }^{3}$ Departamento de Análisis Matemático, Universidad de Sevilla, Apdo. 1160, E-41080 Sevilla, Spain \\ (Received 28 June 2009; revised manuscript received 15 December 2009; published 23 March 2010)
}

\begin{abstract}
Equations describing the evolution of particles, solitons, or localized structures, driven by a zero-average, periodic, external force, and invariant under time reversal and a half-period time shift, exhibit a ratchet current when the driving force breaks these symmetries. The biharmonic force $f(t)=\epsilon_{1} \cos \left(q \omega t+\phi_{1}\right)+\epsilon_{2} \cos (p \omega t$ $+\phi_{2}$ ) does it for almost any choice of $\phi_{1}$ and $\phi_{2}$, provided $p$ and $q$ are two coprime integers such that $p+q$ is odd. It has been widely observed, in experiments in semiconductors, in Josephson junctions, photonic crystals, etc., as well as in simulations, that the ratchet current induced by this force has the shape $v \propto \epsilon_{1}^{p} \epsilon_{2}^{q} \cos \left(p \phi_{1}\right.$ $\left.-q \phi_{2}+\theta_{0}\right)$ for small amplitudes, where $\theta_{0}$ depends on the damping $\left(\theta_{0}=\pi / 2\right.$ if there is no damping, and $\theta_{0}$ $=0$ for overdamped systems). We rigorously prove that this precise shape can be obtained solely from the broken symmetries of the system and is independent of the details of the equation describing the system.
\end{abstract}

DOI: 10.1103/PhysRevE.81.030102

PACS number(s): 05.60.Cd, 05.45.Yv

Ratchetlike transport phenomena, where a net motion of particles or solitons is induced by zero-average forces, can be observed in many physical systems. Such is, for instance, the dc current in semiconductors [1-3], the net motion of fluxon in long Josephson junctions (JJs) [4,5], of vortices in superconductors [6], of cold atoms in optical lattices $[7,8]$, or the rectificatio of Brownian motion [9-11]. In some of these systems, the ratchetlike motion is induced by means of spatial asymmetries $[12,13]$. In the others the transport can also appear if some temporal symmetries are broken by timedependent forces, e.g., [13-18]. This latter case has two advantages: it is generally easier to analyze theoretically, and it is more amenable to experimental observation, e.g., in semiconductors [2], in JJs [4,5], or in optical lattices $[8,19]$.

A large number of simulations and experiments have show $[2,4,5,7,8,11,20-27]$ that in many different systems the behavior of the ratchet velocity $v$ driven by the $T$-periodic biharmonic force

$$
f(t)=\epsilon_{1} \cos \left(q \omega t+\phi_{1}\right)+\epsilon_{2} \cos \left(p \omega t+\phi_{2}\right),
$$

where $T=2 \pi / \omega, \phi_{1}$ and $\phi_{2}$ are the phases, $p$ and $q$ are coprimes with $p+q$ odd, and the amplitudes $\epsilon_{1}$ and $\epsilon_{2}$ are small, is given by the expression

$$
v=B e_{1}^{p} \epsilon_{2}^{q} \cos \left(p \phi_{1}-q \phi_{2}+\theta_{0}\right),
$$

where $B$ and $\theta_{0}$ depend on the parameters of the model and on $\omega$ but neither on the amplitudes nor on the phases $[4,8,17,19,22,23,26]$. It has also been shown for specifi systems that nondissipative dynamics have $\theta_{0}=\pi / 2[7,15]$, whereas overdamped ones have $\theta_{0}=0[5,21,27]$. The aim of this Rapid Communication is to show that symmetry considerations alone are enough to predict behavior (2). This is a

\footnotetext{
*niurka@us.es

†cuesta@math.uc3m.es

tran@us.es
}

strong result because it is valid for any equation that describes the system, no matter the type of nonlinear terms it may contain, as long as it shows invariance under certain symmetry transformations - which will state precisely below.

Attempts at determining the shape of current (2) can be found even in the pioneering works $[2,20]$, aimed at developing a sensitive method of measuring deviations from Ohm's law. Their analysis, however, relies on an expansion of $v$ in odd moments of $f(t)$, justifie by the adiabatic response of the system to an applied fiel (see also [28]). While it cannot be ruled out that such an expansion holds for some systems, or in this adiabatic limit, it is certainly not valid in general. In fact, if one applies that expansion to related dissipative systems, like those of Refs. $[2,4,7,8,15,17,22,23,26]$, the value $\theta_{0}=0$ is always obtained, whereas $\theta_{0} \neq 0$ in general-it can even be $\theta_{0}=\pi / 2$ when dissipation vanishes. We illustrate this fact by analyzing in the supplementary material [38] an exactly solvable example. Therefore one can readily see that the moment expansion is in general an incorrect assumption; only in the overdamped or the adiabatic limits could this expansion become correct, but we do not know of any proof that this holds for systems other than this specifi example.

Let $E[x(t), f(t)]=0$ denote a functional equation (which can represent an ordinary or partial differential equation, an integral equation, etc.) describing the evolution of a particle, soliton, or localized structure whose position is given by $x(t)$, under the driving of a zero-average, external, periodic force $f(t)=f(t+T), T>0$. One such system is said to have ratchetlike behavior if the average velocity, define as [13]

$$
v=\lim _{t \rightarrow \infty} \frac{1}{t} \int_{0}^{t} \dot{x}(\tau) d \tau=\lim _{t \rightarrow \infty} \frac{x(t)}{t},
$$

independent of the initial conditions [29], is nonzero. Consider two temporal transformations: time reversal $(\mathcal{R}: t \mapsto$ $-t)$ and time shift $(\mathcal{S}: t \mapsto t+T / 2)$, and suppose that their action on the force $f(t)$ is given by 


$$
\begin{gathered}
(\mathcal{R} f)(t)=f(-t)=f(t), \\
(\mathcal{S} f)(t)=f(t+T / 2)=-f(t) .
\end{gathered}
$$

Suppose further that any of these transformations - with the appropriate transformation of $x(t)$-leaves $E[x(t), f(t)]=0$ invariant. Nondissipative systems provide typical examples of this kind of behavior such as the equation of motions of cold atoms in optical lattices [7], the dynamic of a particle in a symmetric potential [15], and the soliton ratchets in the extended systems $[17,25]$.

For these kind of systems and forces which satisfy either Eq. (4) or (5) - or both - there can be no ratchet effect because any of the two transformations changes the sign of $v$ ( $\mathcal{R}$ because time goes backward, and $\mathcal{S}$, because $f$ changes sign). As a matter of fact, this is a nice illustration of Curie's principle [30].

In some other cases, time reversal leaves the equation invariant provided the force transforms itself as

$$
(\mathcal{R} f)(t)=f(-t)=-f(t)
$$

instead of Eq. (4). The most prominent examples of this are equations describing overdamped systems such as the vortex motion in JJs [5], the overdamped Brownian motion [16], and the ratchet dynamics of breathers in the discrete Schrödinger equation [27].

Again, and for the same reason, no ratchet effect can appear if the driving force fulfill Eq. (6). In this case, however, in general breaking symmetries (5) and (6) is not enough to induce a ratchet current, some additive noise is necessary as well [21].

Whichever the case, a biharmonic force such as Eq. (1) is able to break both Eq. (4) [or Eq. (6)] and Eq. (5) and induce a ratchet current. In what follows we will prove that, provided a ratchet current is produced, the symmetries impose that it be of form (2).

Let us begin by noticing that $v$ must be a functional of $f(t)$, which we can expand as

$$
\begin{gathered}
v[f]=v_{0}+\sum_{n=1}^{\infty} v_{n}[f], \\
v_{n}[f]=\left\langle c_{n}\left(t_{1}, \ldots, t_{n}\right) f\left(t_{1}\right) \cdots f\left(t_{n}\right)\right\rangle,
\end{gathered}
$$

where $\langle X\rangle \equiv T^{-n} \int_{0}^{T} d t_{1} \cdots \int_{0}^{T} d t_{n} X$ and $v_{0}=v[0]$. This functional Taylor expansion is a rigorous result of functional analysis valid for a very wide class of functionals on Banach spaces (see [31-33] for details). As $v[-f]=-v[f]$ for any force $f(t)$, $c_{2 n}\left(t_{1}, \ldots, t_{2 n}\right) \equiv 0$, so only odd terms appear in expansion (7). On the other hand, the functions $c_{n}\left(t_{1}, \ldots, t_{n}\right)$ can be taken $T$ periodic in each variable and can always be chosen totally symmetric under any exchange of their arguments. Notice in passing that only if $c_{n}\left(t_{1}, \ldots, t_{n}\right) \propto \delta\left(t_{1}\right.$ $\left.-t_{2}\right) \cdots \delta\left(t_{n-1}-t_{n}\right)$ can $v$ be expanded in moments of $f(t)$ - thus the moment expansion is only a particular case of Eq. (7).

Let us now specialize Eq. (7) for biharmonic force (1). First of all, $v$ is not affected by the choice of time origin; thus $v\left[\mathcal{T}_{\tau} f\right]=v[f]$, where $\left(\mathcal{T}_{\tau} f\right)(t)=f(t+\tau)$ for any $\tau$. But $f(t$ $+\tau)=\epsilon_{1} \cos \left(q t+\widetilde{\phi}_{1}\right)+\epsilon_{2} \cos \left(p t+\widetilde{\phi}_{2}\right)$, with $\widetilde{\phi}_{1}=\phi_{1}+q \tau$ and $\widetilde{\phi}_{2}=\phi_{2}+p \tau$, so $v[f]$ must depend on the phases only through the combination $\theta=p \phi_{1}-q \phi_{2}=p \widetilde{\phi}_{1}-q \widetilde{\phi}_{2}$.

Now we must compute $v_{n}[f]$ for any odd $n>0$. By expanding Eq. (1) in complex exponentials,

$$
v_{n}[f]=\sum_{|\mathbf{n}|=n, \mathbf{n} \geq \mathbf{0}} A(\mathbf{n}) \epsilon_{1}^{n_{1}+n_{2}} \epsilon_{2}^{n_{3}+n_{4}} e^{i\left[\left(n_{1}-n_{2}\right) \phi_{1}+\left(n_{3}-n_{4}\right) \phi_{2}\right]},
$$

where $\mathbf{n}=\left(n_{1}, n_{2}, n_{3}, n_{4}\right),|\mathbf{n}| \equiv n_{1}+n_{2}+n_{3}+n_{4}$, and $\mathbf{n} \geq \mathbf{0}$ denotes a componentwise inequality. Besides, because of the symmetry of the functions $c_{n}\left(t_{1}, \ldots, t_{n}\right)$,

$$
\begin{aligned}
A(\mathbf{n})= & \frac{n ! 2^{-n}}{4}\left\langle c_{n}\left(t_{1}, \ldots, t_{n}\right) e^{i \omega \mathbf{v} \cdot\left(t_{1}, \ldots, t_{n}\right)}\right\rangle, \\
& \prod_{i=1} n_{i} !
\end{aligned}
$$

where

$$
\mathbf{v} \equiv(\overbrace{q, \ldots, q,-q, \ldots,-q}^{n_{1}}, \overbrace{p, \ldots, p}^{n_{2}}, \overbrace{-p, \ldots,-p}^{n_{3}}) .
$$

The complex number $A(\mathbf{n})=B(\mathbf{n}) e^{i \psi(\mathbf{n})}$, where

$$
\begin{gathered}
\psi\left(n_{1}, n_{2}, n_{3}, n_{4}\right)=-\psi\left(n_{2}, n_{1}, n_{4}, n_{3}\right), \\
B\left(n_{1}, n_{2}, n_{3}, n_{4}\right)=B\left(n_{2}, n_{1}, n_{4}, n_{3}\right) .
\end{gathered}
$$

Let us focus now on the complex exponential in Eq. (8). We know that $v$ must be a function of $\theta$, so the only nonzero terms in this sum are those satisfying $\left(n_{1}-n_{2}\right) \phi_{1}+\left(n_{3}\right.$ $\left.-n_{4}\right) \phi_{2}=k \theta$ for some $k \in \mathbb{Z}$, i.e., $n_{1}-n_{2}=k p$ and $n_{4}-n_{3}=k q$. But that means $|k|(p+q)=\left|n_{1}-n_{2}-n_{3}+n_{4}\right| \leq\left|n_{1}+n_{2}+n_{3}+n_{4}\right|$ $=n$.

Suppose $n<p+q$; then $k=0$, which implies $n_{1}=n_{2}$ and $n_{3}=n_{4}$, and therefore $n$ must be even. Thus $v_{n}[f]$ must be zero for any odd $n<p+q$, but since there are no even terms in Eq. (7), this means that no term with $n<p+q$ contributes to $v$.

Suppose now $n \geq p+q$; then $|k|>0$ ( $k=0$ is excluded because it would lead to an even $n$ ) and so there will be nonzero terms in Eq. (8) corresponding to powers $\epsilon_{1}^{2 n_{2}+|k| p} \epsilon_{2}^{2 n_{3}+|k| q}$ or $\epsilon_{1}^{2 n_{1}+|k| p} \epsilon_{2}^{2 n_{4}+|k| q}$. The lowest order is $n=p+q$, and is obtained either when $n_{2}=n_{3}=0, n_{1}=p$, and $n_{4}=q$ (i.e., $k=1$ ), or when $n_{1}=n_{4}=0, n_{2}=p$, and $n_{3}=q$ (i.e., $k=-1$ ). Because of Eq. (10), the contribution of these two terms to Eq. (8) is

$$
v_{p+q}=B \epsilon_{1}^{p} \epsilon_{2}^{q} \cos \left(\theta+\theta_{0}\right),
$$

where $B=2 B(p, 0,0, q)$ and $\theta_{0}=\psi(p, 0,0, q)$.

Let us now assume that the equation is invariant when the force satisfie (4). Then, since $\left\langle c_{n}\left(t_{1}, \ldots, t_{n}\right) f\left(-t_{1}\right) \cdots f\left(-t_{n}\right)\right\rangle$ $=\left\langle c_{n}\left(-t_{1}, \ldots,-t_{n}\right) f\left(t_{1}\right) \cdots f\left(t_{n}\right)\right\rangle$, and $v[\mathcal{R} f]=-v[f]$, hence

$$
c_{n}\left(-t_{1}, \ldots,-t_{n}\right)=-c_{n}\left(t_{1}, \ldots, t_{n}\right) .
$$

Applied to Eq. (9) this means that $B(\mathbf{n}) e^{-i \psi(\mathbf{n})}=-B(\mathbf{n}) e^{i \psi(\mathbf{n})}$; i.e., $\psi(\mathbf{n})=\pi / 2$ for all $\mathbf{n}$.

On the other hand, if the equation is invariant when the force satisfie Eq. (6), then $v[-\mathcal{R} f]=-v[f]$, so (recall that $n$ is odd) 


$$
c_{n}\left(-t_{1}, \ldots,-t_{n}\right)=c_{n}\left(t_{1}, \ldots, t_{n}\right) .
$$

Applied to Eq. (9) this means that $B(\mathbf{n}) e^{-i \psi(\mathbf{n})}=B(\mathbf{n}) e^{i \psi(\mathbf{n})}$; i.e., $\psi(\mathbf{n})=0$ for all $\mathbf{n}$.

What we have just shown is that the mean velocity, $v$, of the ratchet current induced by biharmonic force (1) in an equation which is invariant under Eq. (5) always has form (2) if the amplitudes $\epsilon_{1}$ and $\epsilon_{2}$ are small. The coefficient $B$ and $\theta_{0}$ depend on the frequency and on the remaining parameters of the system but not in a universal way that can be predicted under symmetry arguments such as these ones. This shape for the current has been observed, mostly for $p$ $=2$ and $q=1$, in experimental, numerical, and theoretical results in several seemingly unrelated systems $[4,7,15,17,21-23,25]$. For $p=4$ and $q=1$, the collective coordinate on soliton ratchets developed in [26] also confirm Eq. (2).

If the equation is also invariant under Eq. (4) $[7,15]$, then $\theta_{0}=\pi / 2$ and we recover the form $v \sim \epsilon_{1}^{p} \epsilon_{2}^{q} \sin \theta$, whereas if the equation is invariant under Eq. (6), then $\theta_{0}=0$ and $v$ $\sim \epsilon_{1}^{p} \epsilon_{2}^{q} \cos \theta$, in agreement with the vortex motion observed in $\mathrm{JJS}_{\mathrm{S}}$ [5], with the overdamped stochastic dynamic of particles studied in [11,21], and with the ratchet mobility of breathers in the discrete nonlinear Schrödinger equation computed for $p=2$ and $q=1$ in [27].

Notice that formula (2) does not imply that $B$ must be nonzero (Curie's principle). It only proves, under symmetry arguments, that the leading term of $v$ can be of that precise form. It might well happen, for some specifi equation, that
$B=0$. In this case this analysis shows that the leading term must have a dependence on the amplitudes through powers higher than $p$ and $q$. It is likely that if this occurs it will be the fingerprin of a hidden symmetry which, properly broken, will restore result (2).

This analysis provides a direct way to quantitatively relate the causes and the consequences of phenomena through $\mathrm{Cu}$ rie's principle. For instance, our study can be extended to the so-called gating effect, i.e., when the amplitude of spatial or fiel potentials for particles or solitons, respectively, is modifie by a multiplicative force $g(t)=\epsilon_{2} \cos \left(p \omega t+\phi_{2}\right)$ as well as an additive force, $f(t)=\epsilon_{1} \cos \left(q \omega t+\phi_{1}\right)$, with $p$ and $q$ coprimes, acts on the system [34-36]. In such systems, if both $f(t)$ and $g(t)$ satisfy Eq. (4) (in the nondamped limit) or Eq. (6) (in the overdamped limit), or $f(t)$ fulfill Eq. (5), a ratchet transport cannot be induced. A similar procedure shows that, when these symmetries are broken, the average ratchet velocity is also given by Eq. (2), where $\theta_{0}=0$ or $\theta_{0}$ $=\pi / 2$ in the nondamped or overdamped limits, respectively [37].

We acknowledge financia support through Grant Nos. MTM2006-13000-C03-01 and MTM2009-12740-C03-02 (R.A.N.), No. FIS2008-02380/FIS (N.R.Q.), and MOSAICO (J.A.C.), from Ministerio de Educación y Ciencia, Spain; Grant Nos. FQM262 (R.A.N.), No. FQM207 (N.R.Q.), and No. P06-FQM-01735 (N.R.Q. and R.A.N.), from Junta de Andalucía, Spain; and project MODELICO-CM (J.A.C.) from Comunidad de Madrid, Spain.
[1] H. J. Carlin and Y. K. Pozhela, Proc. IEEE 53, 1788 (1965).

[2] W. Schneider and K. Seeger, Appl. Phys. Lett. 8, 133 (1966).

[3] K. N. Alekseev, M. V. Erementchouk, and F. V. Kusmartsev, Europhys. Lett. 47, 595 (1999).

[4] A. V. Ustinov, C. Coqui, A. Kemp, Y. Zolotaryuk, and M. Salerno, Phys. Rev. Lett. 93, 087001 (2004).

[5] S. Ooi, S. Savel'ev, M. B. Gaifullin, T. Mochiku, K. Hirata, and F. Nori, Phys. Rev. Lett. 99, 207003 (2007).

[6] J. E. Villegas, S. Savel'ev, F. Nori, E. M. González, J. V. Anguita, R. García, and J. L. Vicent, Science 302, 1188 (2003).

[7] M. Schiavoni, L. Sánchez-Palencia, F. Renzoni, and G. Grynberg, Phys. Rev. Lett. 90, 094101 (2003).

[8] R. Gommers, S. Bergamini, and F. Renzoni, Phys. Rev. Lett. 95, 073003 (2005).

[9] M. O. Magnasco, Phys. Rev. Lett. 71, 1477 (1993).

[10] P. Reimann and P. Hänggi, Appl. Phys. A: Mater. Sci. Process. 75, 169 (2002).

[11] P. Hänggi, F. Marchesoni, and F. Nori, Ann. Phys. 14, 51 (2005).

[12] R. D. Astumian and P. Hänggi, Phys. Today 55(11), 33 (2002).

[13] P. Reimann, Phys. Rep. 361, 57 (2002).

[14] A. Ajdari, D. Mukamel, L. Peliti, and J. Prost, J. Phys. I 4, 1551 (1994).

[15] S. Flach, O. Yevtushenko, and Y. Zolotaryuk, Phys. Rev. Lett. 84, 2358 (2000).
[16] P. Reimann, Phys. Rev. Lett. 86, 4992 (2001).

[17] M. Salerno and Y. Zolotaryuk, Phys. Rev. E 65, 056603 (2002).

[18] D. Cole, S. Bending, S. Savel'ev, A. Grigorenko, T. Tamegai, and F. Nori, Nature Mater. 5, 305 (2006).

[19] R. Gommers, P. Douglas, S. Bergamini, M. Goonasekera, P. H Jones, and F. Renzoni, Phys. Rev. Lett. 94, 143001 (2005).

[20] C. E. Skov and E. Pearlstein, Rev. Sci. Instrum. 35, 962 (1964).

[21] F. Marchesoni, Phys. Lett. A 119, 221 (1986).

[22] O. Yevtushenko, S. Flach, Y. Zolotaryuk, and A. A. Ovchinnikov, Europhys. Lett. 54, 141 (2001).

[23] A. Engel, H. W. Müller, P. Reimann, and A. Jung, Phys. Rev. Lett. 91, 060602 (2003).

[24] I. Goychuk and P. Hänggi, Europhys. Lett. 43, 503 (1998).

[25] L. Morales-Molina, N. R. Quintero, F. G. Mertens, and A. Sánchez, Phys. Rev. Lett. 91, 234102 (2003).

[26] L. Morales-Molina, R. Niurka Quintero, A. Sánchez, and F. G. Mertens, Chaos 16, 013117 (2006).

[27] A. V. Gorbach, S. Denisov, and S. Flach, Opt. Lett. 31, 1702 (2006).

[28] S. Denisov, S. Flach, A. A. Ovchinnikov, O. Yevtushenko, and Y. Zolotaryuk, Phys. Rev. E 66, 041104 (2002).

[29] The definitio of $v$ is independent on the initial conditions for any dissipative system. Nondissipative systems are idealizations impossible to realize experimentally. They can only be 
QUINTERO, CUESTA, AND ALVAREZ-NODARSE

approached as the limit of very weak damping [8,22]. In this sense, definitio (3) remains valid in this limit.

[30] P. Curie, J. Phys. (Paris), Lett. III, 393 (1894).

[31] R. F. Curtain and A. J. Pritchard, Functional Analysis in Modern Applied Mathematics (Academic Press, London, 1977), theorem 6.4, p. 101.

[32] J. J. Binney, N. J. Dowrick, A. J. Fisher, and M. E. J. Newman, The Theory of Critical Phenomena: An Introduction to the Renormalization Group (Oxford University Press, Oxford, 1992), Appendix L, p. 404.

[33] J. P. Hansen and I. R. McDonald, Theory of Simple Liquids, 3rd ed. (Academic Press, London, 2006), formula 3.2.24, p.
PHYSICAL REVIEW E 81, 030102(R) (2010)

53.

[34] M. Borromeo and F. Marchesoni, Chaos 15, 026110 (2005).

[35] E. Zamora-Sillero, N. R. Quintero, and F. G. Mertens, Phys. Rev. E 74, 046607 (2006).

[36] R. Gommers, V. Lebedev, M. Brown, and F. Renzoni, Phys. Rev. Lett. 100, 040603 (2008).

[37] N. R. Quintero, J. A. Cuesta and R. Álvarez-Nodarse (unpublished).

[38] See supplementary material at http://link.aps.org/supplemental/ 10.1103/PhysRevE.81.030102 for an exactly solvable example. 